\title{
Bremsstrahlung Versus Monoenergetic Photon Dose and Photonuclear Stimulation Comparisons at Long Standoff Distances
}

\section{0th International Conference on Applications of Nuclear Techniques}

The INL is a

U.S. Department of Energy

National Laboratory

operated by

Battelle Energy Alliance

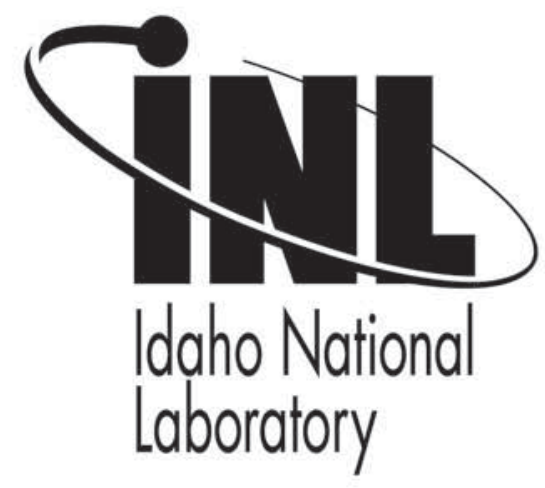

J. L. Jones

J. W. Sterbentz

W. Y. Yoon

D. R. Norman

\section{June 2009}

This is a preprint of a paper intended for publication in a journal or proceedings. Since changes may be made before publication, this preprint should not be cited or reproduced without permission of the author. This document was prepared as an account of work sponsored by an agency of the United States Government. Neither the United States Government nor any agency thereof, or any of their employees, makes any warranty, expressed or implied, or assumes any legal liability or responsibility for any third party's use, or the results of such use, of any information, apparatus, product or process disclosed in this report, or represents that its use by such third party would not infringe privately owned rights. The views expressed in this paper are not necessarily those of the United States Government or the sponsoring agency. 


\title{
Bremsstrahlung versus Monoenergetic Photon Dose and Photonuclear Stimulation Comparisons At Long Standoff Distances
}

\author{
J. L. Jones, J.W. Sterbentz, W.Y. Yoon, D.R. Norman \\ P.O. Box 1625, MS 2802 \\ Idaho Falls, Idaho 83415-2802 \\ james.jones@inl.gov
}

\begin{abstract}
Energetic photon sources with energies greater than $6 \mathrm{MeV}$ continue to be recognized as viable source for various types of inspection applications, especially those related to nuclear and/or explosive material detection. These energetic photons can be produced as a continuum of energies (i.e., bremsstrahlung) or as a set of one or more discrete photon energies (i.e., monoenergetic). This paper will provide a follow-on extension of the photon dose comparison presented at the $\boldsymbol{9}^{\text {th }}$ International Conference on Applications of Nuclear Techniques (June 2008). Our previous paper showed the comparative advantages and disadvantages of the photon doses provided by these two energetic interrogation sources and highlighted the higher energy advantage of the bremsstrahlung source, especially at long standoff distances (i.e., distance from source to the inspected object). This paper will pursue higher energy photon inspection advantage (up to $100 \mathrm{MeV}$ ) by providing dose and stimulated photonuclear interaction predictions in air and for an infinitely dilute interrogated material (used for comparative interaction rate assessments since it excludes material self-shielding) as the interrogation object positioned forward on the inspection beam axis at increasing standoff distances. In addition to the direct energetic photon-induced stimulation, the predictions will identify the importance of secondary downscattered/attenuated source-term effects arising from the photon transport in the intervening air environment.
\end{abstract}

*Supported in part by the Defense Threat Reduction Agency and Department of Energy Idaho Operations Office under Contract Number DE-AC07-05ID14517.

Keywords: Bremsstrahlung, Monoenergetic photons, Photonuclear interactions.

PACS: $24.10 . \mathrm{Ix}, 24.30 . \mathrm{Cz}, 25.20 .-\mathrm{x}, 21.60 . \mathrm{Ka}$

\section{INTRODUCTION}

For over a century ${ }^{1}$ photons have enabled many unique radiographic, medical, and industrial applications, and their interactions in the environment and shielding materials are reasonably well understood and accepted. Photons are readily available using either radioisotopic sources or produced by accelerators. Since higher energy photons can provide significant penetration into inspected objects, as well as inducing material identifiable signatures, energetic $(>6-\mathrm{MeV})$ photon inspection systems continue to be of interest for numerous homeland security and nonproliferation applications, especially for the detection of concealed nuclear materials. These energetic photons can be produced having a broad, continuous energy (bremsstrahlung) or be generated having discrete energies as monoenergetic photons. This paper will focus on complete electron-to-photon production and transport of bremsstrahlung photons and only the transport of isotropically-emitted, monoenergetic 
photons assuming the inspecting gamma-rays have been generated from a protondriven interaction with an assumed proton(ion)-to-photon production efficiency of 1.0; but realizing that actual conversion efficiencies will be many orders of magnitude less than 1.0 depending on the target material and proton energy.

Our previous conference paper ${ }^{2}$ provided an initial photon dose assessment of bremsstrahlung versus monoenergetic photon inspections up to $30 \mathrm{MeV}$ and $1 \mathrm{~km}$ from the photon source (i.e., inspection standoff distance). This paper extends this numerical photon dose assessment (using the general purpose Monte Carlo charge and neutral particle transport code - MCNPX ${ }^{3}$ ) with up to $100-\mathrm{MeV}$ inspection energies, provides photonuclear interaction results of an inspected object, assesses the importance of secondary photon air interactions relative to an inspected object's photonuclear interactions, and finally, provides an air activation assessment of these two energetic photon interrogation techniques.

\section{INSPECTION PHOTON DOSE}

For air at standard temperature and pressure $\left(20^{\circ} \mathrm{C}, 1\right.$ Atm. $)$, Fig. 1 shows the MCNPX-calculated, on-beam axis, bremsstrahlung photon dose rate for 10, 30, 60, and $100-\mathrm{MeV}$ electron beam energies and the isotropic monoenergetic photon inspection dose rate for the corresponding interrogating photon energies as a function of standoff distance from the source. The calculated doses are presented in units of rem per minute per microampere of (average) beam current for the starting particle (an electron for bremsstrahlung and a unity-efficiency proton for monoenergetic photons). For bremsstrahlung sources, the standoff distance is defined along the beamline axis and corresponds to the maximum dose rate production. All dose predictions have less than $5 \%$ statistical error.

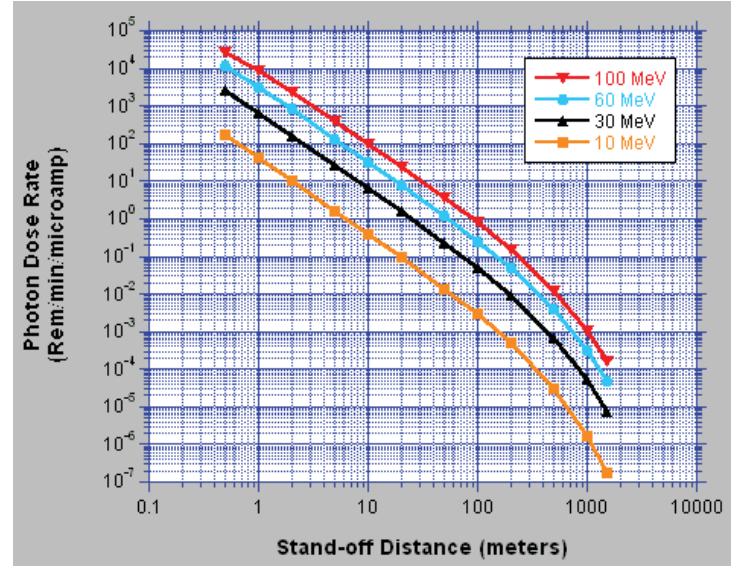

(a)

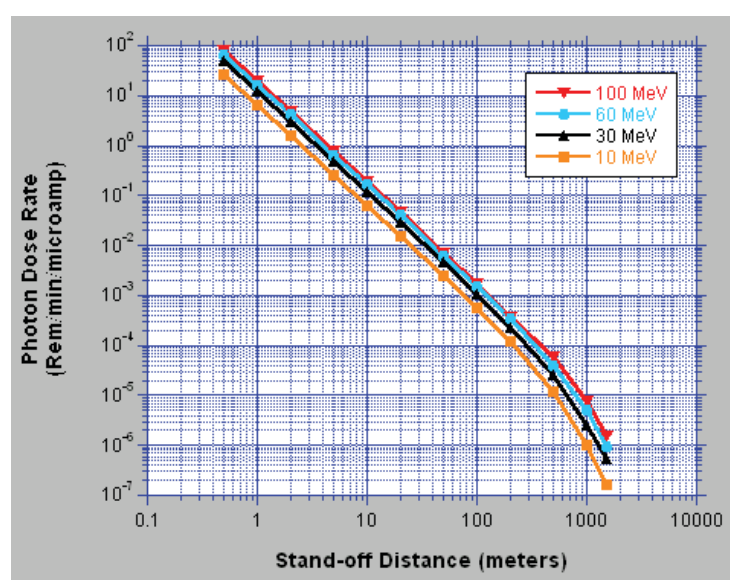

(b)

FIGURE 1. Bremsstrahlung photon dose rate (a) along the inspecting beam axis and monoenergetic photon dose rate (b) at a given distance from the source.

For the bremsstrahlung dose data, the electron-to-photon converter consists of tungsten that varies in thickness to maintain optimal photon production depending on the electron energy, specifically: $0.25,0.62,0.84$, and $1.02 \mathrm{~cm}$ for the $10,30,60$, and $100 \mathrm{MeV}$ electron energies, respectively. The monoenergetic dose results are simply 
based on isotropic, point source emissions of selected gamma-ray energies. The MCNPX photon source models assume no ground plane nor any source collimation and are normalized to a "starting source particle." Any direct comparisons between monoenergetic and bremsstrahlung inspections must account for the actual nuclearreaction production efficiency (i.e., not unity) associated with the proton beam giving rise to the monoenergetic photon production. These monoenergetic results may also be applicable to other non-nuclear reaction methods for generating monoenergetic photons.

As expected, the bremsstrahlung photon dose rate increases (up to two orders of magnitude) with increasing electron beam energies (10-100 MeV) and decreases (up to nine orders of magnitude) with increasing standoff distance (up to $1 \mathrm{~km}$ ). The bremsstrahlung dose profile spread with electron beam energy is primarily due to the nonlinear response in photon source yields with increasing electron energies and the use of optimal photon-yield, electron-photon converter designs.

The monoenergetic photon inspections tend to produce similar (within an order of magnitude) dose rate profile responses for photon interrogations up $100 \mathrm{MeV}$. These latter results follow from known air attenuation cross section behavior that is effectively constant in this photon energy range. Lastly, both monoenergetic and bremsstrahlung interrogations show dose rate profile inflections starting at standoff distances of about $400 \mathrm{~m}$.

\section{PHOTONUCLEAR INTERACTIONS IN AN INSPECTED OBJECT}

To compare bremsstrahlung and isotropic monoenergetic photon interrogation effectiveness using photon interrogation up to $1 \mathrm{~km}$ and $100 \mathrm{MeV}$, an evaluation of total photonuclear interactions in an inspected object, selected to be lead $(\mathrm{Pb})$, is presented since lead represents a common shield material and will provide sufficient photonuclear interactions for this assessment. Furthermore, to maximize the data usefulness and eliminate any geometrical configuration dependency of the inspected object, the object is considered as an infinitely dilute object (i.e., hence eliminating object-dependent dose build-up effects, self shielding, etc.). A key comparison issue involves the two different initial photon inspection spectra and the subsequent photon energy downscattering effects in the air. The latter is important since the giant dipole resonance (GDR) region dominates the photonuclear interactions of most elements and occurs in the 10 to $20-\mathrm{MeV}$ range of interest.

Tables 1 and 2 present the photonuclear interaction results $\left(\# / \mathrm{cm}^{3} /\right.$ source particle), up to $1 \mathrm{~km}$, for bremsstrahlung and monoenergetic photon inspections, respectively. Included in each table are three assessment scenarios or conditions. The first inspection condition, "AIR," includes STP air and its standard elemental composition. This is a baseline inspection scenario addressing all its nominal photon interaction modes, such as photoelectron, Compton scattering, pair production, and photonuclear interactions. The second inspection condition, the "AIR-no secondary" case, includes the STP air but does not transport secondary electrons generated by an interrogating photon interaction nor any converter transmitted source electrons. Hence, for this case, any energetic photons in the air volume between the source and 
TABLE 1. Bremsstrahlung-induced, photonuclear reactions (relative to a source electron (se)) in $\mathrm{Pb}$ at various standoff distances along the beam axis, electron beam energies, and inspection conditions.

(Statistical errors are less than 5\%)

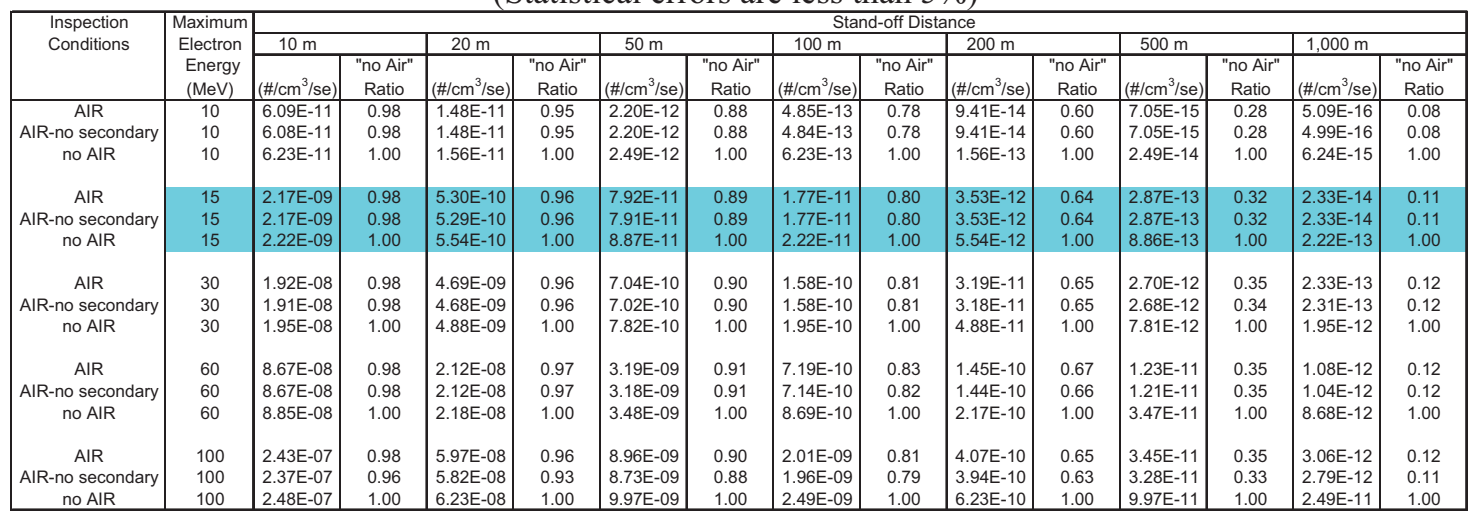

TABLE 2. Monoenergetic-induced, photonuclear reactions relative to a starting source proton (sp) or source photon with unity conversion efficiency in $\mathrm{Pb}$ at various standoff distances along the beam axis, photon beam energies, and inspection conditions. (Statistical errors are less than 5\%.)

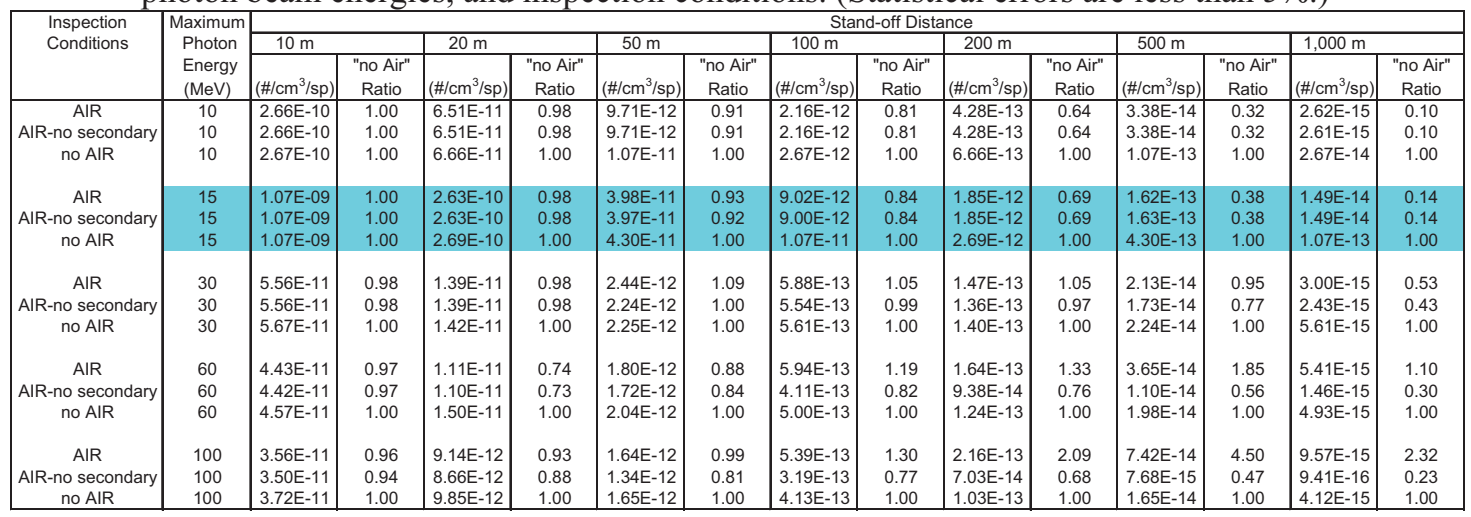

the $\mathrm{Pb}$ object will not produce electrons and for bremsstrahlung inspections, any electrons outside the tungsten converter will not produce photons. This second inspection condition enables the suppression of any subsequent nuclear interactions from pair-produced, energetic secondary positrons and/or electrons in air or in the inspected object at these higher energies. The last condition, "no AIR," removes the air and replaces it with a void; hence, defining a typical $1 / \mathrm{r}^{2}$-isotropic attenuation response with standoff distance. Furthermore, a ratio of interactions to the "no AIR" case for each standoff distance and inspection energy is included in the tables.

The simplest initial comparison involves the voided or "no AIR" inspection scenario since it involves only initial source photon interactions in the inspected object and excludes any energy downscattering effects. Both Table 1 and Table 2 show that in all cases the lead interaction responses follow the expected $1 / \mathrm{r}^{2}$-response dependency (e.g. $(10 / 1000)^{2}=10^{-4}$ ) from the 10 to $1000-\mathrm{m}$ standoff distance. The tables highlight that both $15-\mathrm{MeV}$ interrogations (as well as for lower energy interrogations) appear to show similar overall photonuclear interaction response (within a factor of 2) due to $\mathrm{Pb}$ 's giant dipole resonance interaction region. For monoenergetic inspections less than $15 \mathrm{MeV}$, the interactions decrease since they are 
below the main GDR region and then decrease again with greater monoenergetic inspections since they are now well above the GDR region; indicating that photon energy downscattering effects for monoenergetic photon inspections do not appear to dominate overall interactions yields. Yet, due to the ever-increasing convolution yield of the bremsstrahlung spectra with $\mathrm{Pb}$ 's GDR interaction cross section, the bremsstrahlung interrogations continue to show increasing photonuclear interactions and interrogation standoff ranges with increasing electron beam energies.

The elimination of subsequent radiation effects from secondary particles in air (i.e., the "AIR-no secondary" case) implies that secondary radiation effects with bremsstrahlung inspections appear to have minimal observable impact on the total photonuclear interactions. However, for monoenergetic interrogations, the addition of air appears to support some increased photonuclear interactions (compared to the void case) with larger standoff distances and inspection energies. For a given inspection energy, this trend suggests a build-up of a downscattering mechanism with sufficient standoff distance that may decrease again probably due to attenuation effects related to further increased standoff distances. The elimination of subsequent radiation effects from secondary particles in air (i.e., the "AIR-no secondary" case) appears to support this build-up trend conclusion by showing noticeable photonuclear interaction changes for monoenergetic inspections. Some additional study/benchmarking is warranted to validate and/or better characterize this apparent response with higher monoenergetic inspection energies and distances. When air is included, both photon interrogation types show minimal response differences for standoff distances up to about 100 meters.

\section{AIR ACTIVATION}

For the air activation assessment, Tables 3 and 4 present the results for 30 and 60 $\mathrm{MeV}$ bremsstrahlung endpoint and monoenergetic photon energies, respectively. The assessment uses an uncollimated photon source centered within a 100-m radius, spherical volume. The MCNPX model does not include a ground plane. The calculated results are averaged within the spherical volume and all have statistical errors less than $1 \%$ and typically less than $0.2 \%$. For the bremsstrahlung radiation, the starting source particle is a source electron impacting normally on a tungsten target of thickness $0.62 \mathrm{~cm}$ or $0.84 \mathrm{~cm}$ for the 30 and $60-\mathrm{MeV}$ electrons, respectively, while the monoenergetic radiation is from an assumed, isotropic, point source. Total photonuclear reactions, that include $(\gamma, n),(\gamma, 2 n),(\gamma, 3 n),(\gamma, p)$, and $(\gamma, d)$ cross sections, were calculated for STP air with the major air isotopes: natural nitrogen isotopes $(\mathrm{N}-$ $14, \mathrm{~N}-15)$, oxygen isotopes (O-16, O-17, O-18), carbon isotopes (C-12, C-13), and the one major argon isotope (Ar-40). Table 3 gives the photonuclear bremsstrahlung interactions per cubic centimeter of air per starting source electron (se), whereas similar data is presented in Table 4 for the monoenergetic photon inspections per source photon/proton (sp).

The last column in these tables gives the ratio of the $60 \mathrm{MeV}$ and $30-\mathrm{MeV}$ inspections. The results indicate that the $60-\mathrm{MeV}$ bremsstrahlung beam would increase the air activation products by a factor of approximately 3 to 6 over the $30-\mathrm{MeV}$ bremsstrahlung beam. However, the $60-\mathrm{MeV}$ monoenergetic photon beam would 
produce $20-40 \%$ less air activation products than the $30-\mathrm{MeV}$ monoenergetic beam primarily due to the initial monoenergetic photon spectrum and the limited photon downscattering-effects relative to the air components' photonuclear cross sections.

TABLE 3. Air activation by 30 and $60-\mathrm{MeV}$ bremsstrahlung.

\begin{tabular}{|c|c|c|c|}
\hline Air Isotope & $\begin{array}{c}30-\mathrm{MeV} \mathrm{Inspect.} \\
\left(\text { reactions } / \mathrm{cm}^{3} / \mathrm{se}\right)\end{array}$ & $\begin{array}{c}60-\mathrm{MeV} \mathrm{Inspect.} \\
\left(\text { reactions } / \mathrm{cm}^{3} / \mathrm{se}\right)\end{array}$ & $\begin{array}{c}\text { Ratio } \\
(\text { Brem60/Brem30) }\end{array}$ \\
\hline $\mathrm{N}-14$ & $5.03 \mathrm{E}-17$ & $1.93 \mathrm{E}-16$ & 3.84 \\
\hline $\mathrm{N}-15$ & $2.93 \mathrm{E}-19$ & $9.36 \mathrm{E}-19$ & 3.20 \\
\hline $\mathrm{O}-16$ & $7.46 \mathrm{E}-18$ & $3.87 \mathrm{E}-17$ & 5.18 \\
\hline $\mathrm{O}-17$ & $1.04 \mathrm{E}-20$ & $3.61 \mathrm{E}-20$ & 3.46 \\
\hline $\mathrm{O}-18$ & $7.55 \mathrm{E}-20$ & $2.16 \mathrm{E}-19$ & 2.85 \\
\hline $\mathrm{C}-12$ & $1.97 \mathrm{E}-21$ & $1.22 \mathrm{E}-20$ & 6.21 \\
\hline $\mathrm{C}-13$ & $1.87 \mathrm{E}-22$ & $5.33 \mathrm{E}-22$ & 2.84 \\
\hline $\mathrm{Ar}-40$ & $2.32 \mathrm{E}-18$ & $6.46 \mathrm{E}-18$ & 2.78 \\
\hline
\end{tabular}

TABLE 4. Air activation by 30 and $60 \mathrm{MeV}$ monoenergetic photons.

\begin{tabular}{|c|c|c|c|}
\hline Air Isotope & $\begin{array}{c}30 \mathrm{MeV} \text { Inspect. } \\
\text { (reactions/cm } / \text { sp) }\end{array}$ & $\begin{array}{c}60 \mathrm{MeV} \text { Inspect. } \\
\left(\text { reactions } / \mathrm{cm}^{3} / \mathrm{sp}\right)\end{array}$ & $\begin{array}{c}\text { Ratio } \\
(\text { Mono60/Mono30) }\end{array}$ \\
\hline $\mathrm{N}-14$ & $4.34 \mathrm{E}-16$ & $1.28 \mathrm{E}-16$ & 0.29 \\
\hline $\mathrm{N}-15$ & $1.31 \mathrm{E}-18$ & $4.71 \mathrm{E}-19$ & 0.36 \\
\hline $\mathrm{O}-16$ & $1.24 \mathrm{E}-16$ & $4.66 \mathrm{E}-17$ & 0.38 \\
\hline $\mathrm{O}-17$ & $7.24 \mathrm{E}-20$ & $2.00 \mathrm{E}-20$ & 0.28 \\
\hline $\mathrm{O}-18$ & $4.62 \mathrm{E}-19$ & $8.67 \mathrm{E}-20$ & 0.19 \\
\hline $\mathrm{C}-12$ & $6.45 \mathrm{E}-20$ & $2.24 \mathrm{E}-20$ & 0.35 \\
\hline $\mathrm{C}-13$ & $9.73 \mathrm{E}-22$ & $2.65 \mathrm{E}-22$ & 0.27 \\
\hline $\mathrm{Ar}-40$ & $8.27 \mathrm{E}-18$ & $2.66 \mathrm{E}-18$ & 0.32 \\
\hline
\end{tabular}

Table 5 compares the two inspection energies and source types. The $30-\mathrm{MeV}$ monoenergetic photon source would produce 4-16 times more air activation products than the $30-\mathrm{MeV}$ bremsstrahlung photon source. At $60 \mathrm{MeV}$, the monoenergetic photon source would produce approximately half the activation products relative to the bremsstrahlung source, except in the case of the air isotopes O-16 and C-12. Unfortunately, as shown in the prior section, the advantage of a reduced air activation result for monoenergetic inspections must be tempered since it will also corresponds to a less than optimal photonuclear interactions in an inspected object.

TABLE 5. Relative air activation by 30 and $60-\mathrm{MeV}$ monoenergetic and bremsstrahlung photons.

\begin{tabular}{|c|c|c|}
\hline Air Isotope & $\begin{array}{c}\text { Ratio } \\
\text { (Mono30/Brem30) }\end{array}$ & $\begin{array}{c}\text { Ratio } \\
\text { (Mono60/Brem60) }\end{array}$ \\
\hline $\mathrm{N}-14$ & 8.63 & 0.66 \\
\hline $\mathrm{N}-15$ & 4.48 & 0.50 \\
\hline $\mathrm{O}-16$ & 16.63 & 1.21 \\
\hline $\mathrm{O}-17$ & 6.95 & 0.55 \\
\hline $\mathrm{O}-18$ & 6.12 & 0.40 \\
\hline $\mathrm{C}-12$ & 32.78 & 1.83 \\
\hline $\mathrm{C}-13$ & 5.19 & 0.50 \\
\hline $\mathrm{Ar}-40$ & 3.56 & 0.41 \\
\hline
\end{tabular}

It should also be noted that for bremsstrahlung-based inspections, the analysis here has been based on an average over the 100 m-radius, spherical air volume. In actuality, 
the energetic bremsstrahlung photon radiation is highly forward directed and will result in levels of air activation along the beam line that will be much higher, typically 3 to 4 orders-of-magnitude higher per cubic centimeter per electron, than represented in the averaged tabular data. For the isotropic monoenergetic photon inspections, the average data is representative in all emission angles. Also, the practical existence of a ground plane will greatly reduce air activation concerns for both photon inspection applications.

\section{CONCLUSIONS}

Although one-to-one comparisons between bremsstrahlung and monoenergetic photons are difficult without knowing accelerator-specific data and reaction-specific conversion efficiencies, relative comparisons of dose and interactions can be made on a "per starting source particle" basis. In general, photon interrogation dose rates decrease by more than the inverse-square of the inspection standoff distance primarily due to air attenuation effects. Bremsstrahlung inspection doses are very dependent on the selected electron beam energy, whereas monoenergetic inspection doses are relatively independent of the inspecting 30 to $100-\mathrm{MeV}$ photons.

Photonuclear interactions in an infinitely-dilute $\mathrm{Pb}$ inspection object, calculated using on-beam axis results, are driven by material photonuclear GDR cross sections and appear to show similar (per source particle) results for either $15-\mathrm{MeV}$ bremsstrahlung or monoenergetic inspections; with optimal results for the latter inspection. For bremsstrahlung inspections, the photonuclear interaction yields in an inspected object continue to increase with increasing electron beam energy (suggesting a dose "tuneability" at a selected standoff distance with interaction optimization using the electron beam current and energy), while monoenergetic inspections decrease from an optimal photon energy (effectively corresponding to the general 10-20 MeV GDR region in most elements). In addition, a potential "downscattering-type" interaction enhancement has been identified (comparing the "with and without" AIR scenarios) for high inspection energies and long standoff distances that warrants further investigation.

Finally, if determined to be important for outdoor-type applications, despite the typical wind-driven, air exchange process and the absorption effect of any existing ground plane structure, air activation concerns are less for energetic monoenergetic photons than for bremsstrahlung. However, relative activation issues with higher energy monoenergetic photon inspections approach bremsstrahlung inspection activations but do so with correspondingly lower photonuclear interactions in an inspected object.

This assessment shows that any deployment of energetic photon interrogation techniques must trade-off/optimize issues related to the interrogation dose rate as a function of the inspection distance, the needs related to stimulated interactions in an inspected object, and any potential concerns related to air activation.

\section{REFERENCES}

1. W. C. Röntgen, Phys.-Med. Gesellschaft Würsburg, 132 (1895) 
2. Jones, J.L., et al., "Bremsstrahlung versus Monoenergetic Photons for Photonuclear Inspection Applications, " Proceedings from the $9^{\text {th }}$ International Conference on Applications of Nuclear Techniques, Crete, Greece, June 2008.

3. "MCNPX-A General Purpose Monte Carlo Radiation Transport Code, Version 2.5.0," Los Alamos National Laboratory, New Mexico, April 2005. 